\title{
Grade 4 Liver Laceration after Cardiopulmonary Resuscitation: A Case Report
}

\author{
Sharoon Samuel ${ }^{1^{*}}$, Brent Brown ${ }^{2}$, Nita Mason ${ }^{3}$, Tony Abdo ${ }^{2}$ \\ ${ }^{1}$ Department of Internal Medicine, University of South Dakota, Sanford School of Medicine, Vermillion, United States \\ ${ }^{2}$ Department of Pulmonary \& Critical Care, Oklahoma University Health Sciences Center, Oklahoma City, OK, United States \\ ${ }^{3}$ Department of Radiological Sciences, Oklahoma University Health Sciences Center, Oklahoma City, OK, United States
}

Corresponding Author: Sharoon Samuel, MD

Address: University of South Dakota, Sanford School of Medicine, 1400 W 22nd St Sioux Falls, SD 57104, United States. Tel: (347) 515-4699. Email: sharoon.samuel@usd.edu

Received date: 03 April 2021; Accepted date: 26 April 2021; Published date: 03 May 2021

Citation: Samuel S, Brown B, Mason N, Abdo T. Grade 4 Liver Laceration after Cardiopulmonary Resuscitation: A Case Report. Asp Biomed Clin Case Rep. 2021 May 03;4(2):93-98.

Copyright (C) 2021 Samuel S, Brown B, Mason N, Abdo T. This is an open-access article distributed under the Creative Commons Attribution License, which permits unrestricted use, distribution, and reproduction in any medium provided the original work is properly cited.

\begin{abstract}
Cardiopulmonary resuscitation (CPR) is a life-saving medical procedure used in the setting of cessation of cardiac and respiratory function of a patient to maintain vital functions. CPR was developed in the 1950s as mouth to mouth resuscitation. Defibrillation, chest compression and ventilation were added to CPR in 1960 . Chest compressions are thought to be the most important aspect of CPR and adequate performance of chest compression is required for successful resuscitation as emphasized in recent guidelines. However, chest compressions may be a source of injury to ribs, sternum, spleen, stomach, or liver. We present a case of a 30year-old female with end-stage renal disease, hypertension, polysubstance abuse, and GERD who underwent CPR resulting in a grade 4 liver laceration. The new guidelines of Advanced Cardiac Life Support emphasize the need of regular forceful chest compressions during CPR which, although required for proper resuscitation of the patient may result in significant injuries to the viscera as seen in this patient. The aim of this case report is to highlight the possible complications of CPR and its long-term consequences.
\end{abstract}

\section{Keywords}

Laceration, Cardiopulmonary Resuscitation, Chest Compressions Liver Trauma, Bleeding

\section{Introduction}

Cardiopulmonary resuscitation (CPR) is a medical procedure performed in patients with cardiac arrest. It includes chest compressions, ventilation and sometimes, cardioversion to restore cardiopulmonary function. The chest compressions in adults must be $5.5 \mathrm{~cm}$ or 2 inches in depth which requires about 60 pounds of force. This may result in bodily injury of the patient including ecchymoses of the chest, fracture of the ribs or sternum [1], injury to visceral organs including spleen, stomach, liver [2] and rarely to the heart from rib fracture [3].

We present a case of 30-year-old female with low body mass index (BMI) of $15 \mathrm{~kg} / \mathrm{m}^{2}$ and multiple comorbidities including end stage renal disease (ESRD), hypertension (HTN), polysubstance abuse, osteopenia secondary to ESRD and gastroesophageal reflux disease (GERD) presenting with a grade 4 liver laceration after CPR.

\section{Case Presentation}


Citation: Samuel S, Brown B, Mason N, Abdo T. Grade 4 Liver Laceration after Cardiopulmonary Resuscitation: A Case Report. Asp Biomed Clin Case Rep. 2021 May 03;4(2):93-98.

\section{Case Report}

A 30-year-old female presented to the emergency department with acute onset shortness of breath. She had significant past medical history of ESRD and was on dialysis, also comorbid hypertension, osteopenia secondary to ESRD, polysubstance abuse and GERD. Past medical history included asthma, previous deep venous thromboses (DVTs) and hypothyroidism. Patient also had a low BMI of $15 \mathrm{~kg} / \mathrm{m}^{2}$. Her current medications included hydrocodone/acetaminophen, gabapentin, pantoprazole, promethazine, diphenhydramine, ondansetron, calcium acetate, cholecalciferol, isosorbide mononitrate, metoprolol tartrate, amlodipine, folic acid/vitamin B12, and cinacalcet.

On presentation, the patient had a high blood pressure of $174 / 86 \mathrm{mmHg}$, with all other vitals in normal range. The patient was found to be volume overloaded with bilateral lung crackles and pedal edema, chest $\mathrm{x}$ ray showed pulmonary venous congestion and interstitial edema. Labs showed elevated potassium levels, $8 \mathrm{mg} / \mathrm{dL}$ most likely due to missing dialysis. She was given medications to correct hyperkalemia acutely and transferred to the intensive care unit (ICU). Nephrology was consulted and she was sent to emergent dialysis [4]. She was dialyzed for the volume overload and fluid intake was limited [5]. Post dialysis potassium was down to $3.8 \mathrm{mg} / \mathrm{dL}$. During her $2^{\text {nd }}$ post admission day, the patient had brain natriuretic peptide (BNP) levels of 3,000 which was concerning for congestive cardiac failure [6] and was treated appropriately. After primary treatment, the patient was hemodynamically stable with a Glasgow Coma Scale (GCS) of 14. She was transferred out of the ICU.

On the $5^{\text {th }}$ post admission day, the patient had cardiac arrest and 2ominutes of advanced cardiac life support (ACLS) [7] was performed resulting in the return of spontaneous circulation (ROSC). The patient was transferred back to the ICU for further care. On regaining consciousness in the intensive care unit (ICU) the patient extubated herself. Her oxygen saturation and arterial blood gases were normal on non-invasive ventilation. She did not require repeat intubation. In the ICU, labs showed increasing transaminitis possibly due to shock liver [8].

The following evening the patient's hemoglobin dropped from $9.3 \mathrm{~g} / \mathrm{dL}$ to $6.5 \mathrm{~g} / \mathrm{dL}$ prompting urgent intervention by Interventional Radiology due to suspected intrabdominal bleed. Computed tomography (CT) abdomen with contrast was done which showed a grade 4 liver laceration with a focus of enhancement within the laceration highly concerning for active bleeding (Fig-1a and Fig-1b). The right lobe of the liver also showed a small peripheral laceration on day 1 post CPR with subsequent expansion and active bleeding
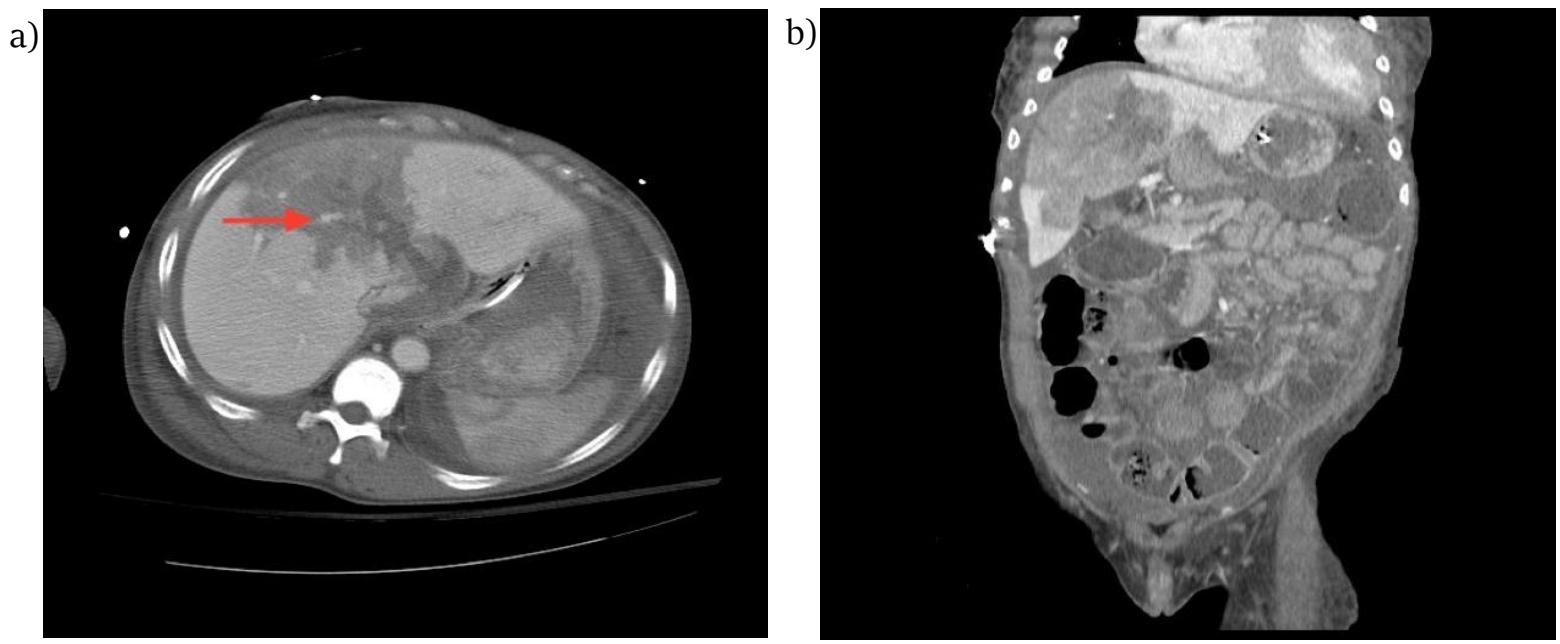

Fig-1a \& 1b:

Axial and coronal post-contrast images of the abdomen with large, grade IV liver laceration. The focus of enhancement within the liver laceration (red arrow) is highly concerning for active bleeding. Additionally, there is noted to be moderate-tolarge volume hemoperitoneum. 
Citation: Samuel S, Brown B, Mason N, Abdo T. Grade 4 Liver Laceration after Cardiopulmonary Resuscitation: A Case Report. Asp Biomed Clin Case Rep. 2021 May 03;4(2):93-98.

\section{Case Report}

a)

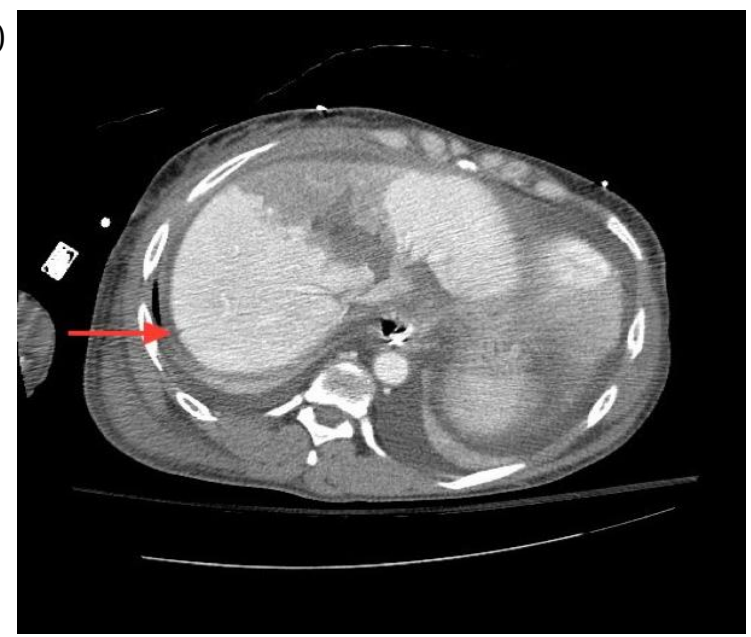

b)

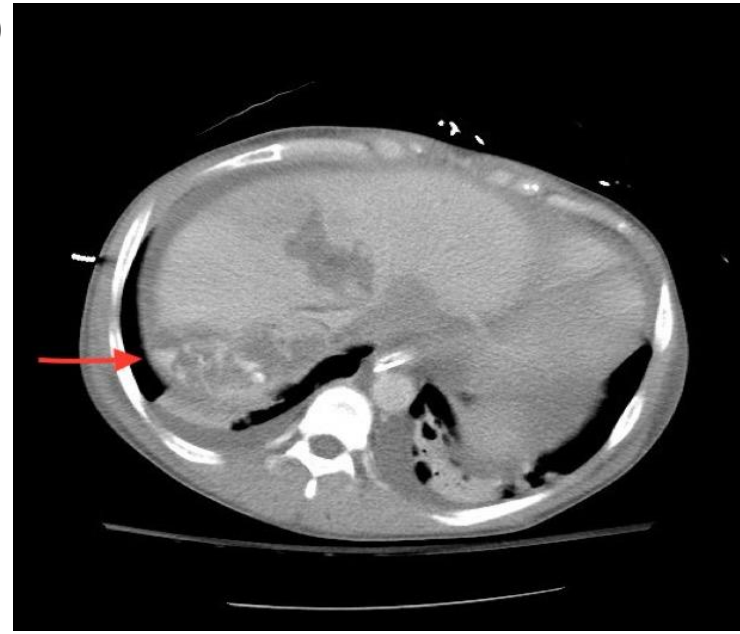

Fig-2a \& 2b:

Axial post contrast images of the abdomen show a small, peripheral laceration in the right lobe of the liver on day 1(Fig. 2a, red arrow) with subsequent expansion and active bleeding on day 2(Fig. 2b, red arrow). Again, shown is the large central liver laceration.

a)

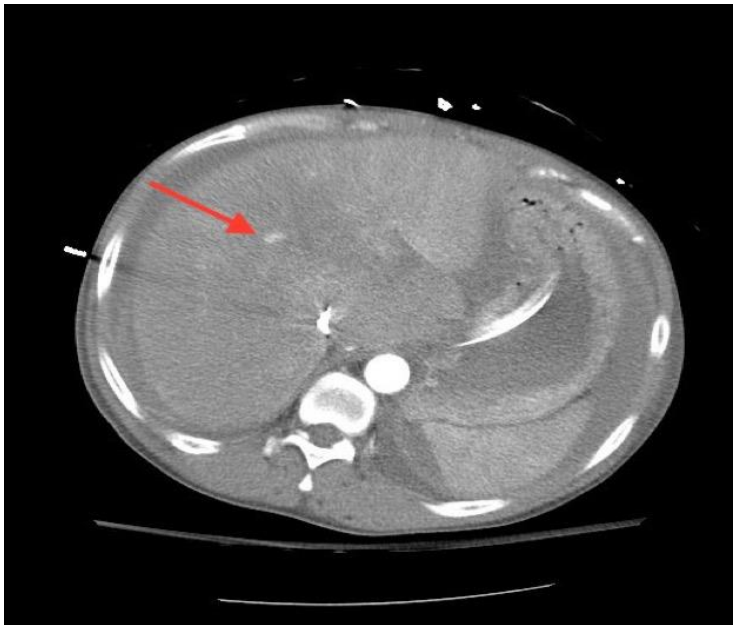

b)

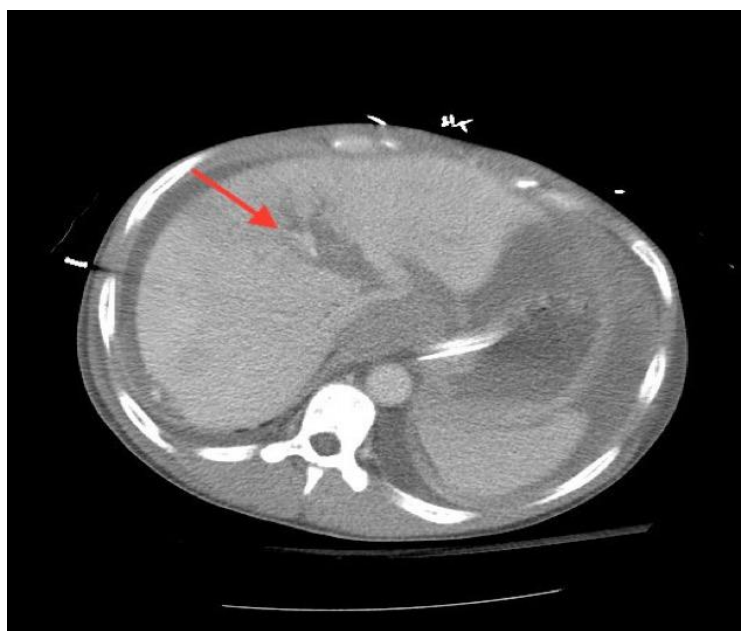

Fig-3a \& $3 \mathrm{~b}:$

Axial post contrast images of the abdomen in arterial (Fig. 3a) and venous (Fig. 3b) phases on day 2 show active arterial bleeding within the large central liver laceration (red arrows).

on day 2 (Fig-2a and Fig-2b). Abdominal post contrast images in arterial and venous phases on day 2 clearly demarcated the source of active bleeding from a large central liver laceration (Fig-3a and Fig-3b). Multiple overlying right and left rib fractures were also noted, most likely due to recent $\mathrm{CPR}$ and underlying osteopenia. The patients rib fractures were essentially not severely displaced and were predominantly in the upper portion of the rib cage. The buckle fractures that were in the more inferior ribs do not show up well in the 3D model (Fig-4, Fig-5 and Fig-6), since we are looking at the ribs from the outside, and the buckles are on the inner table of the ribs.
A large volume hemoperitoneum was also noted. This was a technically difficult study to visualize the hepatic artery as a possible source of the bleed. A CT angiogram was ordered which showed focal heterogeneous enhancement was noted involving segments $4 \mathrm{~A} \& 4 \mathrm{~B}$ with small foci of arterial enhancement. This expanding liver laceration was grade 4 (involving $25-75 \%$ of a hepatic lobe along with parenchymal disruption) and involved segment 7 of the liver with active extravasation. Large hemoperitoneum was also demonstrated. It was planned to perform embolization of left hepatic, middle hepatic and right hepatic arteries by interventional radiology approach. Embolization of the left hepatic, 
Citation: Samuel S, Brown B, Mason N, Abdo T. Grade 4 Liver Laceration after Cardiopulmonary Resuscitation: A Case Report. Asp Biomed Clin Case Rep. 2021 May 03;4(2):93-98.

\section{Case Report}

middle hepatic and right hepatic arteries were performed, the patient required 2 packed cell transfusions due to continued hemoglobin drop.

After stabilization of the hemoglobin, the patient was transferred to medical floors. She was discharged from the hospital after 3 weeks. Careful questioning of the patient's family about traumatic injuries before hospitalization excluded the possible causes prior to admission that would explain the patient's liver laceration.
4)

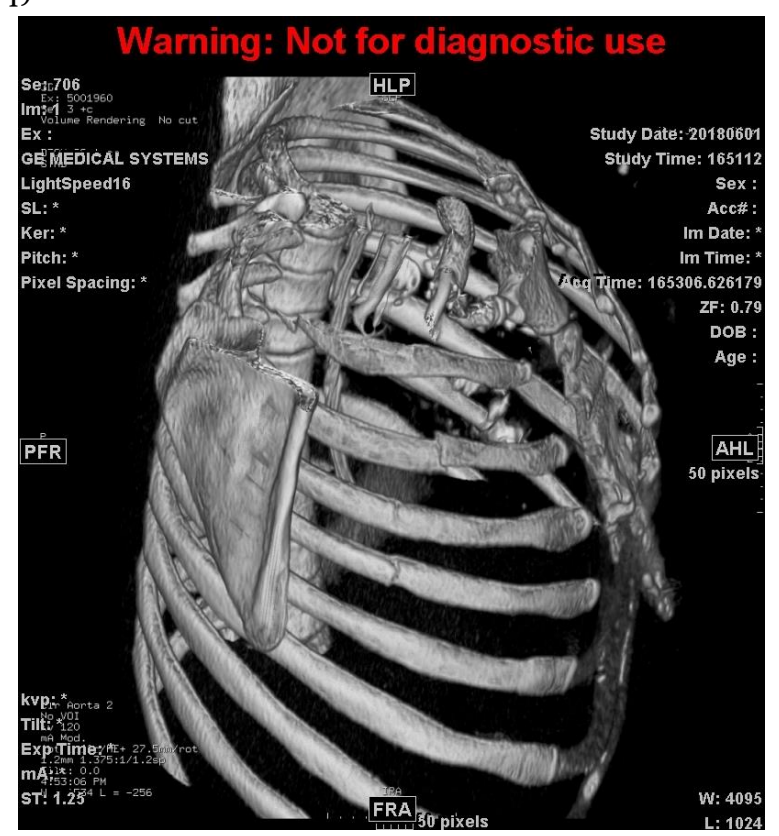

5)

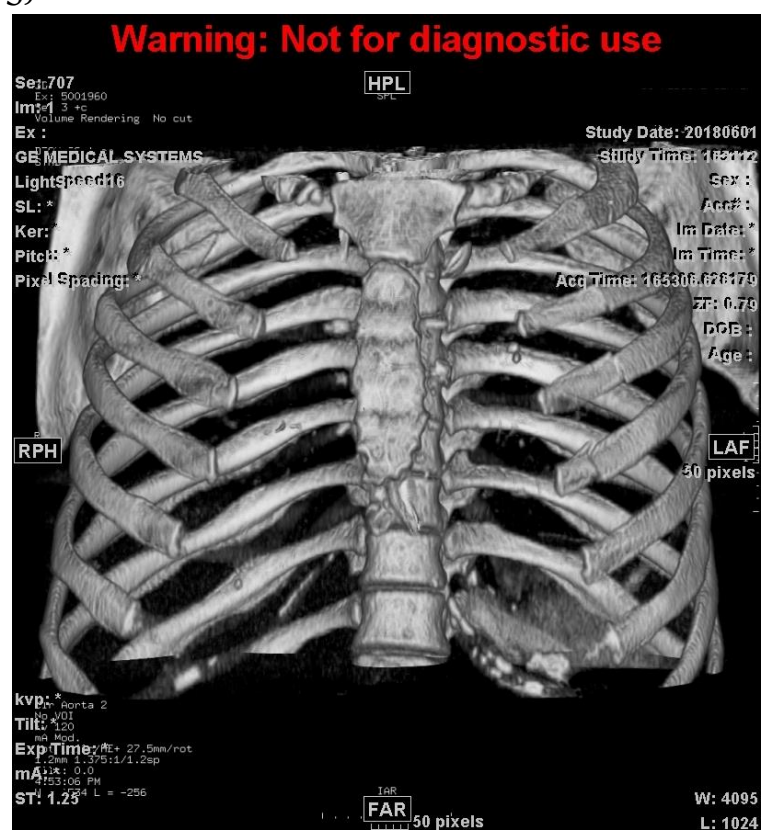

6)

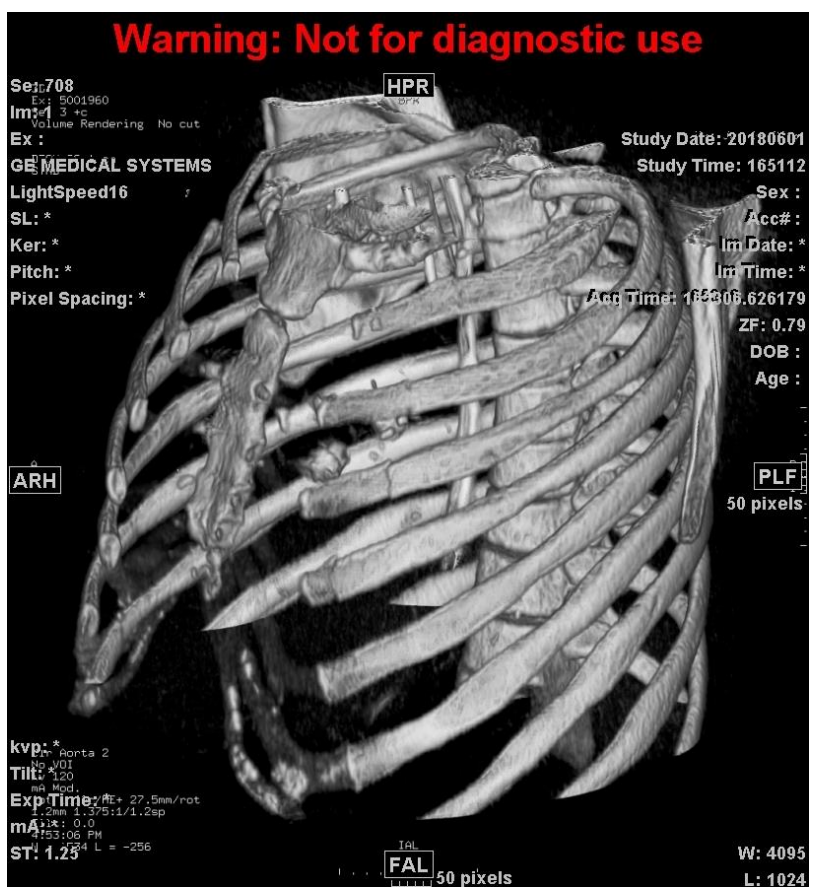

Fig-4,5 \& 6:

Visible rib fractures in the upper portion of the rib cage not severely displaced. Buckle fractures in the inferior portion of the rib cage are not clearly visible in the $3 D$ model as they are in the inner table of the rib. 


\section{Discussion}

Although often lifesaving, CPR can be associated with various types of complications and physical injuries, even when performed in the correct manner as delineated in the literature. The European Resuscitation Council and The American Heart Association recommend chest compression of $4-5 \mathrm{~cm}$ at a rate of $100-120$ beats/min in adults for CPR [9]. Effective CPR can result in increased risk for injuries to intra-abdominal structures [10]. The complications associated with CPR range from ecchymoses, abrasions, hematomas, mucosal injuries of the mouth, injury to teeth, tongue, and larynx, laceration of carotid intima may be associated with airway management. Additionally, skin burns due to defibrillation and cardiac or renal injuries due to rhabdomyolysis can be observed [11].

Although a wide range of injuries may be associated with $\mathrm{CPR}$, the procedure still maintains its critical importance in reviving patients with respiratory and cardiac arrest. Patients with CPR related superficial and external thoracic injuries have good long-term prognosis since these injuries are self-healing if no significant co-morbid factors are involved [12-14]. Patients with visceral organ injuries have increased mortality, especially due to cardiac injuries leading to tamponade, GI bleeding or spleen rupture [9]. A study by Kaldirim et. al. Indicates a positive correlation between the duration of CPR and potentially severe complications of visceral organ damage. There was a statistically significant relation between age and lifethreatening injuries after CPR. The study also found that female patients were predisposed to have more severe injuries after CPR [13].

The most common liver injury due to CPR involves the left liver lobe as seen in this patient. The reason is thought to be the close anatomic proximity of left lobe of liver with the xiphoid process of the sternum. The authors do not believe that the rib fractures in the patient described are the source of the major central liver laceration, which is more of a shear injury (or perhaps just true blunt trauma). Imagine, chest compressions with the hands not placed on the upper sternum, but, more inferiorly closer to the xiphoid or sliding with compression across the patient's right upper abdomen. The patient's low BMI of $15 \mathrm{~kg} / \mathrm{m}^{2}$ and small body habitus in addition to improper hand positioning most likely contributed to the liver laceration.

Due to the significant increase in mortality relating to the visceral injuries caused by CPR, attention should be paid by health care providers to hand positioning, force during compression and pre-existing coagulopathy [15].

Further studies should be published about the longterm prognosis of patients with visceral injuries after CPR. Physicians should be on the look-out for worsening clinical status of the patient after ROSC and major abnormalities in post CPR labs should not be overlooked, especially in older patient population and patients on anticoagulation therapy.

\section{Conclusion}

CPR is a crucial life-saving procedure. When performed according to the recommended guidelines CPR related injuries are still common but it should not impede clinicians in performing proper resuscitation efforts and proper hand placement. Most CPR related Injuries are overlooked. A possibility of CPR related injuries should always be considered in patients who have delayed recovery after return of spontaneous circulation (ROSC).

Automated feedback devices which are used to measure the proper depth of compressions can also help in avoiding repeated deep compressions beyond 2 inches in depth, a possible cause of injuries.

\section{Funding Source}

Authors have no relevant financial relationships to disclose.

\section{Conflicts of Interest}

All authors have read and approved the final version of the manuscript. The authors have no conflicts of interest to declare.

\section{References}

[1] Black CJ, Busuttil A, Robertson C. Chest wall 
injuries following cardiopulmonary resuscitation. Resuscitation. 2004 Dec;63(3):339-43. [PMID: 15582770]

[2] Beydilli H, Balci Y, Erbas M, Acar E, Isik S, Savran

B. Liver laceration related to cardiopulmonary resuscitation. Turk J Emerg Med. 2016 May 9;16(2):7779. [PMID: 27896328]

[3] Machii M, Inaba H, Nakae H, Suzuki I, Tanaka H. Cardiac rupture by penetration of fractured sternum: a rare complication of cardiopulmonary resuscitation. Resuscitation. 2000 Jan;43(2):151-53. [PMID: 10694176]

[4] Weisberg LS. Management of severe hyperkalemia. Crit Care Med. 2008 Dec;36(12):3246-51. [PMID: 18936701]

[5] Claure-Del Granado R, Mehta RL. Fluid overload in the ICU: evaluation and management. BMC Nephrol. 2016 Aug 2;17(1):109. [PMID: 27484681]

[6] Maisel A. B-type natriuretic peptide levels: diagnostic and prognostic in congestive heart failure: what's next? Circulation. 2002 May 21;105(20):232831. Erratum in: Circulation 2002 Jul 16;106(3):387. [PMID: 12021215]

[7] Hallstrom A, Herlitz J, Kajino K, Olasveengen TM. Treatment of asystole and PEA. Resuscitation. 2009 Sep;80(9):975-86. [PMID: 19581035]

[8] Mongardon N, Dumas F, Ricome S, Grimaldi D, Hissem T, Pène F, Cariou A. Postcardiac arrest syndrome: from immediate resuscitation to long-term outcome. Ann Intensive Care. 2011 Nov 3;1(1):45. [PMID: 22053891]
[9] Kim MJ, Park YS, Kim SW, Yoon YS, Lee KR, Lim TH, Lim H, Park HY, Park JM, Chung SP. Chest injury following cardiopulmonary resuscitation: a prospective computed tomography evaluation. Resuscitation. 2013 Mar;84(3):361-64. [PMID: 22819881]

[10] Yang Z, Li H, Yu T, Chen C, Xu J, Chu Y, Zhou T, Jiang L, Huang Z. Quality of chest compressions during compression-only CPR: a comparative analysis following the 2005 and 2010 American Heart Association guidelines. Am J Emerg Med. 2014 Jan;32(1):50-54. [PMID: 24210889]

[11] Hashimoto Y, Moriya F, Furumiya J. Forensic aspects of complications resulting from cardiopulmonary resuscitation. Leg Med (Tokyo). 2007 Mar;9(2):94-99. [PMID: 17276125]

[12] Olds K, Byard RW, Langlois NE. Injuries associated with resuscitation - An overview. J Forensic Leg Med. 2015 Jul;33:39-43. [PMID: 26048495]

[13] Kaldırım U, Toygar M, Karbeyaz K, Arzıman I, Tuncer SK, Eyi YE, et al. Complications of cardiopulmonary resuscitation in non-traumatic cases and factors affecting complications. Egyptian Journal of Forensic Sciences. 2016;6(3):270-74.

[14] Buschmann CT, Tsokos M. Frequent and rare complications of resuscitation attempts. Intensive Care Med. 2009 Mar;35(3):397-404. [PMID: 18807013]

[15] Biswas S, Alpert A, Lyon M, Kaufmann CR. Cardiopulmonary Resuscitation Complicated by Traumatic Hepatic Laceration: A Case Report and Review of Literature. 2017 Mar;8(3):93-97. 